\title{
COVID-19 Precipitating Euglycaemic Diabetic Ketoacidosis with SGLT2 Inhibitor Use
}

\author{
Jiali Fang, Matthew Genco, Rachel N Caskey \\ Department of Medicine, University of Illinois at Chicago, Chicago, USA
}

Received: $12 / 08 / 2020$

Accepted: $14 / 08 / 2020$

Published: $26 / 10 / 2020$

How to cite this article: Fang J, Genco M, Caskey RN. COVID-19 precipitating euglycaenemic diabetic ketoacidosis with SGLT2 inhibitor use. EJCRIM 2020;7: doi:10.12890/2020_001943.

Conflicts of Interests: The Authors declare that there are no competing interests.

This article is licensed under a Commons Attribution Non-Commercial 4.0 License

\section{ABSTRACT}

Objective: To describe a patient who developed euglycaemic diabetic ketoacidosis (DKA) in the setting of SGLT2 inhibitor use precipitated by COVID-19.

Patient and methods: A 52-year-old male with type II diabetes on empagliflozin and no history of DKA presented with symptoms of COVID-19 as well as laboratory findings consistent with euglycaemic DKA. His hospital course was complicated by recurrent episodes of euglycaemic DKA as well as hyperglycaemic DKA.

Conclusion: SGLT2 inhibitors should be held as early as possible in COVID-19 cases due to the risk of euglycaemic DKA. These patients should also have more intense glucose monitoring.

\section{LEARNING POINTS}

- COVID-19 can precipitate euglycaemic DKA in diabetic patients taking SGLT2 inhibitors.

- Clinicians should be cognizant that the effects of SGLT2 inhibitors can persist for more than 72 hours after the last dose.

- Diabetic patients with COVID-19 require closer strict glucose monitoring to reduce the risk of DKA.

\section{KEYWORDS}

Diabetic ketoacidosis, sodium-glucose cotransporter 2 inhibitors, COVID-19, diabetes, empagliflozin

\section{BACKGROUND}

Sodium-glucose cotransporter 2 (SGLT2) inhibitors are a popular class of antihyperglycaemic medications recommended as second-line therapy for type II diabetes after metformin.

Diabetic ketoacidosis (DKA) is a rare life-threatening complication of SGLT2 inhibitor use characterized by hyperglycaemia, metabolic acidosis and ketosis. Studies have shown that up to 71\% of cases of DKA associated with SGLT2 inhibitors are cases of euglycaemic DKA, where serum glucose is $\leq 250 \mathrm{mg} / \mathrm{dl}$, and it is often precipitated by a viral or bacterial infection ${ }^{[1]}$.

In this case study, we will describe a patient who developed euglycaemic DKA in the setting of SGLT2 inhibitor use precipitated by coronavirus disease 2019 (COVID-19).

\section{CASE DESCRIPTION}

A 52-year-old male with type II diabetes diagnosed over 10 years previously hypertension and hyperlipidaemia presented to the emergency room for symptoms of COVID-19. His diabetes was relatively well controlled on metformin and empagliflozin, with the most recent $\mathrm{HbA} 1 \mathrm{c}$ measurement at $8.5 \%$. 
He did not have a history of DKA. His presenting symptoms included decreased oral intake, shortness of breath, cough, fever and diarrhoea. He had not taken any medications for 2 days. On arrival, he was hypoxic to $85 \%$ on room air and the laboratory work-up was concerning for euglycaemic DKA (Table 1). Other causes of anion gap metabolic acidosis were ruled out. The patient was admitted to the intensive care unit and started on an insulin drip and hydration with closure of the anion gap and subsequent transition to basal and bolus insulin 12 hours later. bolus insulin 12 hours later.

The patient's serum glucose levels remained in the low-normal range and bolus/sliding scale insulin was held, which resulted in recurrence of euglycaemic acidosis 8 hours after the gap originally closed. During this recurrence, the patient's urine glucose levels were over 500 mg/ dl while serum glucose values remained at $90-110 \mathrm{mg} / \mathrm{dl}$, probably due to the persistent effects of the empagliflozin taken approximately 4 days prior. The patient was again managed with intravenous hydration and an increased basal-bolus insulin regimen resulting in closure of the anion gap and improvement in beta-hydroxybutyric acid levels.

Unfortunately, his course was complicated by hypoxic respiratory failure from COVID-19 and community-acquired pneumonia requiring intubation a few days later. After intubation, the patient developed worsening acidosis, hypotension and renal failure requiring vasopressors, bicarbonate and brief continuous renal replacement therapy. The patient subsequently developed a third episode of DKA (Table 2) with hyperglycaemia, which was again managed with an insulin drip and hydration. After the anion gap closed, he was transitioned to basal and bolus insulin treatment with stabilization of glucose levels, slow clinical improvement, eventual extubation and no further episodes of DKA.

\begin{tabular}{|l|l|}
\hline Parameter & \\
\hline VBG pH & 7.30 \\
\hline VBG pCO2 & $37 \mathrm{mmHg}$ \\
\hline Aniongap & 18 \\
\hline Venous glucose & $113 \mathrm{mg} / \mathrm{dl}$ \\
\hline Beta-hydroxybutyric acid & $5.0 \mathrm{mmol} / \mathrm{l}$ \\
\hline Urinalysis glucose & $>500 \mathrm{mg} / \mathrm{dl}$ \\
\hline Urinalysis ketones & $80 \mathrm{mg} / \mathrm{dl}$ \\
\hline COVID-19 (nasopharyngeal PCR test) & Detected \\
\hline
\end{tabular}

Table 1. Laboratory findings on presentation to the emergency room

\begin{tabular}{|l|l|}
\hline Parameter: & \\
\hline ABG $p H$ & 7.10 \\
\hline ABG pCO2 & $98 \mathrm{mmHg}$ \\
\hline Anion gap & 23 \\
\hline Venous glucose & $372 \mathrm{mg} / \mathrm{dl}$ \\
\hline Beta-hydroxybutyric acid & $5.4 \mathrm{mmol} / \mathrm{l}$ \\
\hline
\end{tabular}

Table 2. Laboratory findings at the time of the third episode of diabetic ketoacidosis

\section{DISCUSSION}

Although euglycaemic DKA is a known side effect of SGLT2 inhibitors, few clinicians are aware of this complication. Prior to the COVID-19 pandemic, patients were told to discontinue SGLT2 inhibitors before surgery, during acute illness, if they were at any risk of dehydration, poor carbohydrate intake or excessive alcohol intake ${ }^{[1]}$. This recommendation was echoed by guidelines released online in April 2020 by diabetes experts to hold any SGLT2 inhibitors during respiratory infections such as COVID-19, to follow sick day rules and to avoid initiating these medications during respiratory illness ${ }^{[2]}$. Additionally, they recommended that patients with COVID-19 on SGLT2 inhibitors should have more intense monitoring and supportive therapy to reduce the risk of DKA ${ }^{[2]}$.

One of the challenges with these recommendations is the ability of the patient to recognize when they should stop SGLT2 inhibitor use due to the prolonged and variable presentation and progression of COVID-19. Although our patient stopped taking empagliflozin 2 days prior to admission as would have been advised, he still developed euglycaemic DKA both on arrival and a few days into his hospital course. Fortunately, early recognition of euglycaemic DKA prompted appropriate management on admission. Recognizing this atypical presentation of DKA is essential for timely diagnosis and treatment.

Our patient's course was complicated by recurrent episodes of both euglycaemic and hyperglycaemic DKA, possibly from the severity of COVID-19. Though data are currently very limited, 1 study among hospitalized patients in Wuhan, China suggested that COVID-19 can induce DKA in patients with diabetes ${ }^{[3]}$. In our case, the second episode of euglycaemic DKA that occurred 4 days after the patient's last dose points to the prolonged effects of SGLT2 inhibitors, which are longer than previously believed ${ }^{[4]}$. 
As the world searches for potential treatments for COVID-19, SGLT2 inhibitors have also been hypothesized to be a possible treatment option with 2 trials currently enrolling patients ${ }^{[5]}$. The proposed benefits will have to be weighed closely against the risk of euglycaemic DKA.

\section{CONCLUSION}

This unique presentation of euglycaemic DKA in the setting of COVID-19 while on an SGLT2 inhibitor highlights many important features of this medication in the current era. It points to the need for vigilance regarding medication counselling and close monitoring of diabetic patients during this pandemic. It further brings into question the actual length of SGLT2 inhibitor effects and cautions physicians to be aware of the duration of effects. Lastly, it outlines the need for careful observation for this side effect if SGLT2 inhibitors are to be tested as a potential COVID-19 therapy. As our understanding of both COVID-19 and its relationship with SGLT2 inhibitors continues to expand, the medical community must be attentive to this dangerous side effect.

\section{REFERENCES}

1. Goldenberg RM, Berard LD, Cheng AY, Gilbert JD, Verma S, Woo VC, et al. SGLT2 inhibitor-associated diabetic ketoacidosis: clinical review and recommendations for prevention and diagnosis. Clin Ther 2016;38(12):2654-2664.e1.

2. Bornstein SR, Rubino F, Khunti K, Mingrone G, Hopkins D, Birkenfeld AL, et al. Practical recommendations for the management of diabetes in patients with COVID-19. Lancet Diabetes Endocrinol 2020;8(6):546-550.

3. Li J, Wang X, Chen J, Zuo X, Zhang H, Deng A. COVID-19 infection may cause ketosis and ketoacidosis. Diabetes Obes Metab 2020;22:1935-1941.

4. Scheen AJ. Pharmacokinetic and pharmacodynamic profile of empagliflozin, a sodium glucose co-transporter 2 inhibitor. Clin Pharmacokinet 2014;53(3):213-225.

5. Fernandez-Fernandez B, D'Marco L, Górriz JL, Jacobs-Cachá C, Kanbay M, Luis-Lima S, et al. Exploring sodium glucose co-transporter-2 (SGLT2) inhibitors for organ protection in COVID-19. J Clin Med 2020;9(7):2030. 